\title{
Erratum to: Blogging Activities in Higher Education: Comparing Learning Scenarios in Multiple Course Experiences
}

\author{
Elvira Popescu ${ }^{1(\bowtie)}$, Zuzana Kubincová $^{2}$, and Martin Homola ${ }^{2}$ \\ ${ }^{1}$ University of Craiova, Craiova, Romania \\ popescu_elvira@software.ucv.ro \\ ${ }^{2}$ Comenius University in Bratislava, Bratislava, Slovakia \\ \{kubincova, homola\}@fmph. uniba.sk
}

\section{Erratum to: \\ Chapter 18: F.W.B. Li et al. (Eds.) \\ Advances in Web-Based Learning - ICWL 2015 \\ DOI:10.1007/978-3-319-25515-6_18}

The following acknowledgment should have been included in the paper starting on page 197 of this volume:

Acknowledgement. This work was supported by a grant of the Romanian National Authority for Scientific Research and Innovation, CNCS - UEFISCDI, project number PN-II-RU-TE-2014-4-2604. 